\title{
Perfil sociodemográfico e clínico dos pacientes com lesões agudas e crônicas em atendimento ambulatorial
}

\author{
Sociodemographic and clinical profile of patients with acute and chronic injuries in \\ ambulatory care
Perfil sociodemográfico y clínico de pacientes con lesiones agudas y crónicas en atención ambulatoria

Fabiana de Paula Gomes ${ }^{1 \star}$, Nariani Souza Galvão ${ }^{1}$, Aline Duarte Albuquerque ${ }^{1}$.

\begin{abstract}
RESUMO
Objetivo: Analisar as variáveis sociodemográficas e clínicas em pacientes com lesões agudas e/ou crônicas, bem como os fatores demográficos e clínicos associados à sua ocorrência. Métodos: Pesquisa descritiva, transversal, abordagem quantitativa, realizada com usuários de ambos os sexos, cadastrados em um ambulatório de referência no tratamento de feridas na cidade de Manaus-Amazonas, utilizando-se para a coleta de dados um instrumento com dados sociodemográficos e, outro, chamado Bates-Jensen Wound Assessment Tool (BWAT) para avaliação da cicatrização das lesões. Resultados: Encontrou-se predominância de lesões crônicas, localizadas nos membros inferiores e em pacientes do sexo masculino com presença de comorbidades. Evidencia-se a necessidade de traçar estratégias de prevenção, transformação no estilo de vida e atenção aos fatores de risco preveníveis para essas lesões. Os achados poderão ser equiparados com outros estudos em instituições de referência no Brasil, de modo a fornecer subsídios para as políticas públicas de saúde. Conclusão: O enfermeiro destaca-se como parte fundamental no processo de cura das lesões, portanto, deverá obter entendimento necessário sobre os indicadores sociodemográficas e clínicos relacionados aos pacientes com lesões agudas e crônicas para, a partir deste conhecimento, direcionar sua prática assistencial e educacional.
\end{abstract}

Palavras-chave: Enfermagem, Ferimentos e lesões, Assistência Ambulatorial.

\begin{abstract}
Objective: To analyze sociodemographic and clinical variables in patients with acute and / or chronic injuries, as well as demographic and clinical factors associated with their occurrence. Methods: Descriptive, crosssectional research, quantitative approach, carried out with users of both sexes, registered in a reference clinic for the treatment of wounds in the city of Manaus-Amazonas, using an instrument with sociodemographic data for data collection, and another, called Bates-Jensen Wound Assessment Tool (BWAT) for assessing wound healing. Results: There was a predominance of chronic lesions, located in the lower limbs and in male patients with the presence of comorbidities. The need to outline prevention strategies, lifestyle changes and attention to preventable risk factors for these injuries is evident. The findings can be compared with other studies in reference institutions in Brazil, in order to provide subsidies for public health policies. Conclusion: Nurses stand out as a fundamental part in the wound healing process, therefore, they must obtain the necessary understanding of the sociodemographic and clinical indicators related to patients with acute and chronic injuries in order to, based on this knowledge, direct their care and educational practice.
\end{abstract}

Keywords: Nursing, Wounds and injuries, Ambulatory care.

\section{RESUMEN}

Objetivo: Analizar las variables sociodemográficas y clínicas en pacientes con lesiones agudas y / o crónicas, así como los factores demográficos y clínicos asociados a su ocurrencia. Métodos: Investigación descriptiva, transversal, abordaje cuantitativo, realizada con usuarios de ambos sexos, registrados en una clínica de referencia para el tratamiento de heridas en la ciudad de Manaus-Amazonas, utilizando un instrumento con datos sociodemográficos para la recolección de datos, y otro, llamado Herramienta de evaluación de heridas de Bates-Jensen (BWAT) para evaluar la cicatrización de heridas. Resultados: Predominaron las lesiones crónicas, localizadas en miembros inferiores y en pacientes del sexo masculino con presencia de

1 Universidade Federal do Amazonas (UFAM), Manaus - AM. *E-mail: fabianaf245@gmail.com 
comorbilidades. Es evidente la necesidad de delinear estrategias de prevención, cambios en el estilo de vida y atención a los factores de riesgo prevenibles de estas lesiones. Los hallazgos se pueden comparar con otros estudios en instituciones de referencia en Brasil, con el fin de otorgar subsidios a las políticas de salud pública. Conclusión: El enfermero se destaca como parte fundamental en el proceso de cicatrización de heridas, por lo que debe obtener el conocimiento necesario de los indicadores sociodemográficos y clínicos relacionados con los pacientes con lesiones agudas y crónicas para, a partir de este conocimiento, orientar su práctica asistencial y educativa.

Palabras clave: Enfermería, Heridas y traumatismos, Atención ambulatoria.

\section{INTRODUÇÃO}

Uma ferida ou lesão na pele é representada pela interrupção da função e integridade do tecido, causado por agente físico, químico ou biológico. Fatores como traumas mecânicos, químicos, físicos, isquêmicos, e a intenção como, por exemplo, as cirurgias, podem resultar em lesões (LIMA NBA, et al., 2016; MITTAG BF, 2017).

Conforme o tempo de cicatrização dos tecidos, as feridas são classificadas em agudas ou crônicas. Feridas agudas derivam de cirurgias ou traumas e, não ocorrendo nenhum tipo de complicação, a cicatrização transcorre em um intervalo satisfatório de tempo. Devido a complicações, as feridas crônicas não cicatrizam em um intervalo de tempo oportuno, resultado de um processo agudo que respondeu de modo diferente à fase inflamatória da cicatrização (LIMA LV, et al., 2016).

Os tipos mais comuns de feridas crônicas são as lesões vasculares, lesões por pressão e as lesões neuropáticas, originadas em decorrências de doenças crônicas como diabetes e hanseníase, alcoolismo, doenças reumáticas, doenças infecciosas, neoplasias e doenças hematológicas. Muitas destas lesões crônicas são associadas ao fator de idade elevada, menor escolaridade e comprometimento do estado cognitivo (VIEIRA CPB e ARAUJO TME, 2018; VIEIRA CPB, et al., 2017).

Estima-se que nos Estados Unidos da América cerca de 6 milhões de pessoas apresentem feridas crônicas. No Brasil, poucos são os estudos epidemiológicos que permitam designar esse percentual, porém, os dados encontrados da Inglaterra esperam que cerca de 570 mil brasileiros apresentem novas feridas crônicas (VIEIRA CPB e ARAUJO TME, 2019).

Deve-se também considerar a análise clínica, uma vez que a equipe multidisciplinar que prestará assistência ao doente deve conhecer o processo de cicatrização, entre elas identificação das etiologias de base, características histopatológicas e quais suas complicações para que sua assistência seja completa e eficaz (MACEDO EAB, et al., 2019). As lesões agudas subdividem-se em abertas e fechadas atingindo da epiderme, derme, hipoderme, entre outros tecidos. São elas traumáticas, perfurantes, contundentes, por atrito, inoculação de veneno ou toxina de animais, mordeduras, queimaduras, ferida provocada por erisipela e as cirúrgicas que são consideradas feridas agudas intencionais, pois são desempenhadas em centro cirúrgico (CHIBANTE C, et al., 2015; CHAVAGLIA SRR, et al., 2015). Referentes a lesões agudas, os estudos epidemiológicos feitos no Brasil, apontam uma prevalência de lesão por fricção (LF) de 3,3\% em esfera hospitalar (GRDEN CRB, et al., 2018).

Estudos epidemiológicos relacionados a portadores de lesões agudas e crônicas atendidos em ambulatório são ainda escassos nacionalmente, mas principalmente na cidade de Manaus (GALVÃO NS, et al., 2015). Logo demonstra-se a importância de se identificar o perfil dos pacientes e a ocorrência das lesões agudas e crônicas em serviços de atendimento ambulatorial em Manaus para possibilitar que o enfermeiro conheça os fatores associados ao surgimento destas lesões que em sua maioria estão relacionadas às doenças crônicas e contribua no desenvolvimento de ações de prevenção voltadas para a população em todos os níveis de atenção à saúde, principalmente, com ações educativas com orientação voltada para o autocuidado destes indivíduos.

Entender os dados sociodemográficos e saber classificar as lesões da população atendida contribui para que a enfermagem atue de modo eficiente na diminuição do tempo de cicatrização destas lesões, impactando, 
consequentemente, na redução do número de amputações, das sequelas físicas e emocionais que acometem os pacientes. No Amazonas, são poucos os dados tanto sobre a incidência tanto quanto o perfil das pessoas com lesões agudas e crônicas, apesar da demanda de pacientes nos serviços ambulatoriais de referência no Estado. A análise de variáveis clínicas, uma vez que a equipe multidisciplinar que prestará assistência ao doente deve conhecer 0 processo de cicatrização, características histopatológicas, identificação das etiologias de base e quais suas complicações para que sua assistência seja eficaz (OLIVEIRA AC, et al., 2019).

As lesões agudas e crônicas causam grande impacto social e emocional, uma vez que restringem seus portadores de atividades simples diárias e de lazer, dessa forma, a intervenção de enfermagem deve considerar os diversos aspectos do indivíduo, como características sociodemográficas (idade, sexo, doença de base, renda, ocupação, estado civil, nível de escolaridade, entre outras) para direcionar sua comunicação com o paciente, de modo que o mesmo receba suporte emocional e informacional, destacando a prevenção e complicações possíveis (MELO AF, et al., 2019; AZEVEDO IC, et al., 2018).

Para que a equipe de saúde venha a intervir de forma adequada no tratamento das lesões agudas e crônicas, torna-se importante estudar as variáveis sociais e demográficas dos usuários, para que haja um direcionamento no tratamento, linguagem, além de análise da necessidade de apoio social ao paciente (OLIVEIRA AC, et al., 2019). No Brasil, as feridas acometem a população de forma geral, independentemente de sexo, idade ou etnia, determinando alto índice de pessoas com alterações na integridade da pele, constituindo assim um grave problema de saúde pública (LIBERATO SMD, et al., 2017).

Estudo realizado com idosos atendidos nos serviços de atenção primária em capital da região Nordeste do Brasil que encontrou prevalência e etiologia de $8 \%$ com feridas crônicas, com destaque para as lesões por pressão $(5 \%)$ e as feridas venosas $(2,9 \%)$. A presença de ferida crônica está associada às idades mais avançadas, menor escolaridade e comprometimento do estado cognitivo (VIEIRA CPB, et al., 2017). Estudo feito em hospitais no Estado do Rio Grande do Norte evidenciou maior número de queimaduras na faixa etária entre 1 a 4 anos com 30,8\% (571) e 12,2\% (227) dos casos na faixa de 20 a 29 anos de idade. O menor número de queimaduras foi na faixa etária de 80 anos e mais 2,4\% (45) (GALVÃO NS, 2016).

O presente estudo é relevante, pois o mesmo contribuirá com a divulgação de informações a respeito da situação atual das variáveis clínicas e sociodemográficas das lesões agudas e crônicas, que apesar da existência de literaturas nacionais sobre o assunto, ainda são escassas na região norte, além disso, fornecerá subsídios, para a realização de outras pesquisas acerca deste assunto. Nesse contexto, a proposta deste estudo científico visa analisar as variáveis sociodemográficas e clínicas em pacientes portadores de lesões agudas e crônicas, bem como, os fatores demográficos e clínicos associados à sua ocorrência.

\section{MÉTODOS}

Pesquisa descritiva, transversal e quantitativa. Nos estudos descritivos os fatos são observados, registrados, analisados, classificados e interpretados, de modo que não haja intervenção do pesquisador, sendo utilizados procedimentos padronizados para coletar os dados da pesquisa (questionário/entrevista e observação sistemática).

O estudo foi realizado em uma instituição de ensino/assistência, ou seja, hospital-escola que presta serviços de assistência à saúde, em unidade ambulatorial no setor de estomaterapia. A amostra foi constituída por todos os pacientes cadastrados no setor ambulatorial de estomaterapia no período de coleta, constituída por 12 pacientes, respeitando-se os critérios de inclusão. Os critérios de inclusão da amostra foram: possuir lesões agudas e crônicas em tratamento no ambulatório; ter idade acima de 18 anos. $O$ critério de exclusão da amostra foi o abandono do tratamento ou morte.

Foram usados dois instrumentos para a coleta de dados, um sociodemográfico o qual possui perguntas abertas e de múltipla escolha (idade, sexo, raça, estado civil, filhos, ocupação, renda mensal, doenças de base, escolaridade, etilismo, tabagismo, tempo da úlcera e recidivas). O outro chamado de Bates Jensen Wound Assessment Tool adaptado para a língua portuguesa, o instrumento consiste nos seguintes 
parâmetros (área da ferida, aparência do leito e quantidade de exsudato) e é utilizado para avaliar o processo cicatricial da ferida e os resultados das intervenções (ALVES DFS, et al., 2015).

Este instrumento é comumente utilizado para avaliar lesões por pressão, tendo sido aprovado pela organização norte-americana National Pressure Injury Advisory Panel (NPIAP), porém, neste estudo, adaptou-se para o uso nas lesões agudas e crônicas, tendo sido comprovada sua eficácia e confiabilidade para avaliação nas demais úlceras crônicas.

A coleta dos dados foi realizada no período de maio de 2019, através da aplicação dos instrumentos de coleta de dados supracitados e após aprovação do Projeto pelo Comitê de Ética em Pesquisa com Seres Humanos (CEP), parecer de número 3.274.395. Os dados foram tabulados em planilha do Excel e repassados ao software estatístico para a realização da associação entre os dados.

Após esclarecimento sobre os objetivos do estudo, os sujeitos da pesquisa que aceitaram voluntariamente a participar na pesquisa, a assinaram do termo de Consentimento Livre e Esclarecido (TCLE) conforme a Resolução № 466 de 12 de dezembro de 2012 do Conselho Nacional de Saúde (CNS) onde assegura o anonimato, o sigilo dos dados coletados, direito de declinar do estudo em qualquer momento sem penalização.

\section{RESULTADOS E DISCUSSÃO}

\section{Caracterização demográfica e clínica da amostra.}

A amostra caracterizou-se por predomínio do sexo masculino (7/58,33\%), idade média de 59,83 anos (DP $=15,8 \%)$, raça parda $(11 / 91,67 \%)$ e aposentado (7/58,33\%) (Tabela 1). Com relação a estilo de vida da amostra total, constata-se que (8/66,67\%), apresentaram comorbidade como hipertensão arterial, além de hábitos relacionados ao etilismo onde $(5 / 41,67 \%)$ não usa mais álcool e $(5 / 41,67 \%)$ nunca usaram álcool; e tabagismo $(7 / 58,33 \%)$ nunca fizeram uso.

Referente as lesões recidivantes, (6/50\%) não voltaram a abrir, e (6/50\%) voltaram a abrir após cicatrizadas. A categoria de medicamentos com maior predomínio foi de Anti-hipertensivo/Antagonista (7/58,33\%; $p=0,00379)$ (Tabela 1).

Podemos observar que as lesões agudas e crônicas, se localizaram mais frequentemente nos pés D/E $10(75,00 \%)$, com maioria de forma irregular $6(50,00 \%)$, seguido de circular/oval $5(41,67 \%)$, apresentando tamanho menor que $4 \mathrm{~cm}^{2} 5$ (45,45\%). Com relação a profundidade da lesão, houve resultado semelhante quanto ao eritema não branqueável e perda parcial da espessura da pele envolvendo epiderme e/ ou derme, $12(100 \%)$, as margens da lesão mostraram-se definidas em 6 (50\%). Houve resultado semelhante com relação ao descolamento das margens, menor que $<2 \mathrm{~cm}$ em qualquer área e ausência de descolamento 12(100\%) (Tabela 2).

Nenhuma lesão apresentou tecido e quantidade necrótico no leito da ferida, 12 (100\%). Quanto a presença de exsudato, a maioria das lesões não apresentaram (7/58,33\%), enquanto (3/25\%) apresentaram exsudato seroso e, por fim, serossanguinolento em $(2 / 16,67 \%)$ das lesões.

Quanto a quantidade de exsudato, a maioria apresentou, ausentes/feridas secas, e pequenas $8(66,66 \%)$, seguidas de escassa, ferida úmida/moderada 4 (33,34\%). Referente a cor da pele ao redor da ferida, a maioria das lesões foram hipopigmentadas 5 (41,67\%), seguidas da cor rósea 4 (33,33\%).

A maioria das lesões apresentou edema não depressível $<4 \mathrm{~cm}$ ao redor da ferida $5(41,67 \%)$, seguida de edema depressível $<4 \mathrm{~cm}$ ao redor da ferida $4(33,33 \%)$. Quanto ao endurecimento do tecido periférico, 7 $(58,33 \%)$ das lesões foram ausentes, e $3(25,00 \%)<2 \mathrm{~cm}$.

O tecido de granulação estava presente em pele íntegras/tecido vermelho escuro puro em 10 (83,34\%) das lesões, seguidas de $2(16,67 \%)$ em $75 \%$ a $100 \%$ do tecido em ferida preenchida. A epitelização dos tecidos ocorreu em $100 \%$ da ferida coberta, superfície intacta, $7(50 \%)$, seguida de $50 \%$ da ferida $4(32,13 \%)$. 
Tabela 1 - Perfil sociodemográfico dos pacientes com lesões agudas e crônicas.

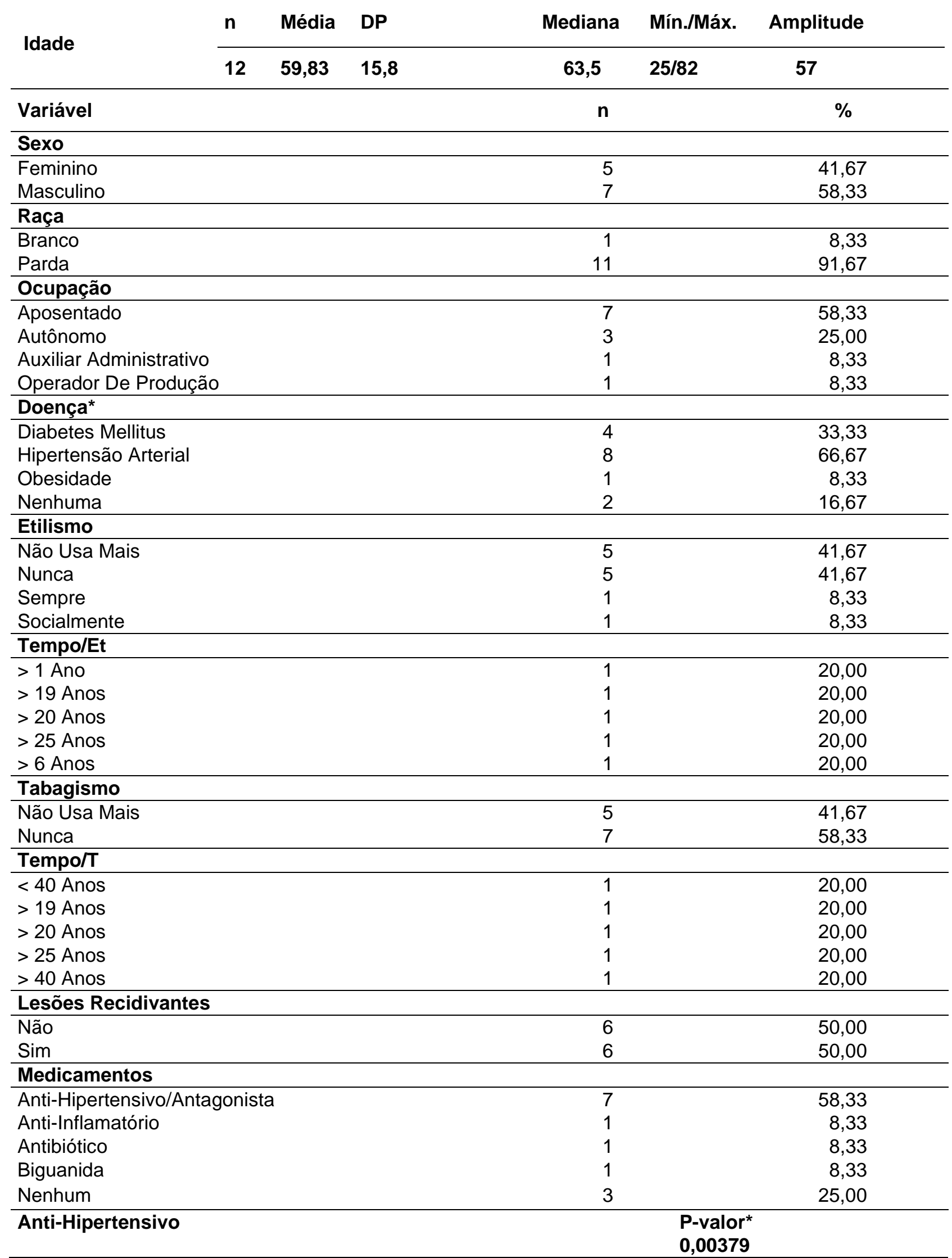

Legenda: *Características clínicas das feridas agudas e crônicas dos pacientes da amostra.

Fonte: Gomes FP, et al., 2020. 
Tabela 2 - Distribuição das lesões agudas e crônicas de acordo com as características clínicas.

\begin{tabular}{|c|c|c|}
\hline Variável & $\mathbf{n}$ & $\%$ \\
\hline \multicolumn{3}{|l|}{ Localização da lesão } \\
\hline $\begin{array}{l}\text { Pé D/E } \\
\text { Outra parte do corpo }\end{array}$ & $\begin{array}{r}10 \\
2\end{array}$ & $\begin{array}{l}75 \\
25\end{array}$ \\
\hline \multicolumn{3}{|l|}{ Forma da lesão } \\
\hline $\begin{array}{l}\text { Circular/oval } \\
\text { Irregular } \\
\text { Linear/ Formato de Borboleta }\end{array}$ & $\begin{array}{l}5 \\
6 \\
1 \\
\end{array}$ & $\begin{array}{c}41,67 \\
50 \\
8,33\end{array}$ \\
\hline \multicolumn{3}{|l|}{ Tamanho } \\
\hline $\begin{array}{l}>80 \mathrm{~cm}^{2} \\
<4 \mathrm{~cm}^{2} \\
16,1-<36 \mathrm{~cm}^{2} / 36,1-<80 \mathrm{~cm}^{2}\end{array}$ & $\begin{array}{l}2 \\
6 \\
4\end{array}$ & $\begin{array}{l}18,18 \\
45,45 \\
36,36\end{array}$ \\
\hline \multicolumn{3}{|l|}{ Profundidade } \\
\hline $\begin{array}{l}\text { Eritema não branqueável/Perda parcial da espessura da pele envolvendo } \\
\text { epiderme e/ou derme }\end{array}$ & 12 & 100 \\
\hline \multicolumn{3}{|l|}{ Margem } \\
\hline $\begin{array}{l}\text { Bem definidas, não aderidas } \\
\text { Definidas } \\
\text { Indefinidas, não visível claramente }\end{array}$ & $\begin{array}{l}1 \\
6 \\
5\end{array}$ & $\begin{array}{c}8,33 \\
50 \\
41,67\end{array}$ \\
\hline \multicolumn{3}{|l|}{ Descolamento } \\
\hline$<2 \mathrm{~cm}$ em qualquer área/ Ausente & 12 & 100 \\
\hline \multicolumn{3}{|l|}{ Tipo de tecido necrótico } \\
\hline Ausente & 12 & 100 \\
\hline \multicolumn{3}{|l|}{ Quantidade de tecido necrótico } \\
\hline Ausente & 12 & 100 \\
\hline \multicolumn{3}{|l|}{ Tipo de exsudato } \\
\hline Ausente & 7 & 58,33 \\
\hline Seroso & 3 & 25 \\
\hline Serossanguinolento & 2 & 16,67 \\
\hline \multicolumn{3}{|l|}{ Quantidade de exsudato } \\
\hline Ausente, ferida seca/ Pequena & 8 & 66,66 \\
\hline Escassa, ferida úmida, mas sem evidência de exsudato/Moderada & 4 & 33,34 \\
\hline \multicolumn{3}{|l|}{ Cor da pele ao redor da ferida } \\
\hline Hiperpigmentada & 1 & 8,33 \\
\hline Hipopigmentada & 5 & 41,67 \\
\hline Rósea & 4 & 33,33 \\
\hline Vermelha brilhante e/ou esbranquiçada ao toque/ Vermelha escura & 2 & 16,66 \\
\hline \multicolumn{3}{|l|}{ Edema do tecido periférico } \\
\hline Edema depressível $<4 \mathrm{~cm}$ a redor da ferida & 4 & 33,33 \\
\hline Edema Não depressível $<4 \mathrm{~cm}$ a redor da ferida & 5 & 41,67 \\
\hline Edema Não depressível $>4 \mathrm{~cm}$ a redor da ferida & 1 & 8,33 \\
\hline Sem edema & 2 & 16,67 \\
\hline \multicolumn{3}{|l|}{ Endurecimento do tecido periférico } \\
\hline$<2 \mathrm{~cm}$ & 3 & 25 \\
\hline $2-4 \mathrm{~cm}$ & 2 & 16,67 \\
\hline Ausente & 7 & 58,33 \\
\hline \multicolumn{3}{|l|}{ Tecido de Granulação } \\
\hline $75 \%$ a $100 \%$ da ferida preenchida & 2 & 16,67 \\
\hline Pele integra/ Vermelho escuro opaco & 10 & 83,34 \\
\hline \multicolumn{3}{|l|}{ Epitelização } \\
\hline $75 \%$ da ferida $/ 25 \%$ da ferida & 4 & 32,13 \\
\hline $50 \%$ da ferida & 1 & 17,87 \\
\hline $100 \%$ da ferida coberta, superfície intacta & 7 & 50 \\
\hline
\end{tabular}

Fonte: Gomes FP, et al., 2020. 
Constata-se a predominância das lesões crônicas em 7 (58,33\%) dos pacientes, seguidos de 5 (41,67\%) pessoas com lesões agudas (Tabela $\mathbf{3}$ ).

Tabela 3 - Distribuição das lesões agudas e crônicas, segundo a classificação.

\begin{tabular}{lll}
\hline Variável & $\mathbf{n}$ & $\%$ \\
\hline Tipo de lesão & & \\
\hline Lesão aguda & 5 & 41,67 \\
Lesão crônica & 7 & 58,33 \\
\hline
\end{tabular}

Fonte: Gomes FP, et al., 2020.

Detectou-se diferença estatisticamente significativa entre as variáveis, cicatrização da lesão e tempo da lesão $(p=0,001)$; cicatrização e recidiva da lesão $(p=0,013)$; cicatrização da lesão e hipertensão arterial $(p=0,0247$ ) (Tabela 4) (Tabela 5).

Tabela 4 - Distribuição quanto ao sexo, tabagismo e recidiva da ferida, segundo a cicatrização da lesão.

\begin{tabular}{|c|c|c|c|c|c|c|c|}
\hline \multirow[b]{2}{*}{ Variável } & \multicolumn{6}{|c|}{ Cicatrização da Lesão } & \multirow[b]{2}{*}{ P-valor } \\
\hline & $\mathbf{n}$ & Média & Mediana & $\begin{array}{l}\text { Desvio } \\
\text { Padrão }\end{array}$ & Mínimo & Máximo & \\
\hline \multicolumn{8}{|l|}{ Sexo } \\
\hline Feminino & 5 & 21,40 & 20 & 11,327 & 9 & 39 & \multirow{2}{*}{0,290} \\
\hline Masculino & 7 & 27,57 & 30 & 8,039 & 17 & 39 & \\
\hline \multicolumn{8}{|l|}{ Tabagismo } \\
\hline Não Usa Mais & 5 & 27,20 & 30 & 6,419 & 17 & 33 & \multirow{2}{*}{0,515} \\
\hline Nunca & 7 & 23,43 & 20 & 11,588 & 9 & 39 & \\
\hline \multicolumn{8}{|l|}{ Recidiva } \\
\hline Sim & 6 & 31,83 & 32 & 6,524 & 24 & 39 & \multirow{2}{*}{0,013} \\
\hline \multirow[t]{2}{*}{ Não } & 6 & 18,17 & 17,5 & 6,911 & 9 & 30 & \\
\hline & \multicolumn{2}{|c|}{${ }^{*} r$} & P-valor & \multicolumn{2}{|l|}{${ }^{* *} \rho$} & \multicolumn{2}{|l|}{ P-valor } \\
\hline Tempo da Lesão (anos) & \multicolumn{2}{|l|}{0,788} & 0,002 & \multicolumn{2}{|c|}{0,840} & 0,001 & \\
\hline
\end{tabular}

Tabela 5 - Distribuição dos pacientes com lesões agudas e crônicas e morbidades clínicas, segundo a cicatrização da lesão. Manaus, 2019.

\begin{tabular}{|c|c|c|c|c|c|c|c|}
\hline \multirow{2}{*}{ Variável } & \multicolumn{6}{|c|}{ Cicatrização da Lesão } & \multirow{2}{*}{ P-valor* } \\
\hline & $\mathbf{n}$ & Média & Mediana & DP & Mínimo & Máximo & \\
\hline \multicolumn{8}{|c|}{ Hipertensão Arterial } \\
\hline Não & 4 & 16,8 & 16,5 & 6,65 & 9 & 25 & \multirow{2}{*}{0,0247} \\
\hline Sim & 8 & 29,1 & 30,5 & 8,2 & 17 & 39 & \\
\hline \multicolumn{8}{|c|}{ Diabetes Mellitus } \\
\hline Não & 8 & 27,1 & 27,5 & 7,66 & 15 & 39 & 0,41 \\
\hline
\end{tabular}

Legenda: * $\mathrm{DP}=$ desvio padrão; *Teste de Mann-Whitney.

Fonte: Gomes FP, et al., 2020.

Pode-se observar que o aparecimento de feridas agudas e crônicas teve maior ocorrência nos homens (7/58,33\%), com idade média de 59,83 anos ( $\mathrm{DP}=15,8 \%$ ) e aposentados $(7 / 58,33 \%)$. O processo natural do envelhecimento promove um aumento na deposição de gordura e modificação no calibre dos vasos, consequentemente, indivíduos com mais de 60 anos possuem um maior número de condições crônicas degenerativas, afetando diretamente o resultado não evolutivo das feridas relaciona-se muitas vezes pelo fato do portador da lesão possuir comorbidade Hipertensão Arterial $(8 / 66,67)$, pois a diminuição na perfusão tissular dificulta o processo de cicatrização dessas feridas. Lesões agudas e crônicas estão relacionadas com aspectos imunológicos do indivíduo e, a existência de patologias crônicas, corrobora de modo negativo no processo de cicatrização de feridas (LIMA BE, et al., 2018). 
Destaca-se a necessidade do tratamento adequado das feridas e do controle de doenças crônica como a hipertensão arterial. Antagonicamente, a insuficiência arterial periférica está diretamente associada à não cicatrização dos tecidos e retardo no fechamento da lesão, portanto, devemos salientar a importância da verificação da pressão arterial.

No presente estudo observou-se que os participantes da pesquisa já possuíram hábitos como tabagismo e etilismo em certo momento de suas vidas, um dos malefícios do tabagismo é a vasoconstrição, afetando o processo cicatricial. As substâncias nocivas liberadas pelo cigarro no sistema nervoso central fazem com que a norepinefrina atue nas terminações nervosas e nos receptores $\alpha$ ocasionando vasoconstrição (LIBERATO SMD, 2017). Dentre os fármacos usados pelos indivíduos participantes do estudo, $(7 / 58,33 \%)$ foi composta por Anti-hipertensivos/Antagonista, seguido de Anti-inflamatório (1/8,33\%), Antibiótico (1/8,33\%), Biguanida $(1 / 8,33 \%)$ e nenhum medicamento $(3 / 25,00 \%)$. As ações destas drogas alteram a fase inflamatória da cicatrização de feridas pois interferem no estágio inicial da inflamação e na produção de fibroblastos, prejudicando a síntese de proteínas e, consequentemente, comprometendo a epitelização.

Com relação ao tempo das lesões, obteve-se que muitos pacientes as possuíam há vários anos em relação com a cicatrização da lesão e tempo da lesão $(p=0,001)$, este achado pode estar associado com o tipo de tratamento de ferida realizado pelos profissionais e, ainda pode estar relacionado com a falta de tratamento, considerando as diretrizes clínicas na prevenção e tratamento de lesões, que contribuem para melhora da assistência prestada aos pacientes (LIMA BE, et al., 2018). Observou-se a importância do papel de pacientes e acompanhantes nos cuidados diários de lesões agudas e crônicas, onde os curativos eram feitos por profissional qualificado em Estomaterapia, juntamente com técnico de enfermagem, além das orientações dadas sobre $o$ autocuidado a ser realizado em casa.

A terapêutica direcionada somente na lesão, esquecendo-se os fatores sistêmicos que interferem na cicatrização, dentre eles as condições vasculares do paciente, corrobora com as recorrências e o tempo da lesão aumentado (VIEIRA CPB, et al., 2017). Apesar de que metade dos participantes desta pesquisa apresentou recidiva $(p=0,013)$, é considerado quantitativo alto pois $100 \%$ dos participantes fazem tratamento continuo com profissionais especializados e produtos adequados.

Um dos principais motivos da recorrência das lesões é a negligência do paciente sobre os cuidados e as medidas de prevenção, por exemplo, pacientes portadores de úlcera venosa que tiveram feridas cicatrizadas após anos sem uso de meias compressivas, displicentemente voltam a ter lesões. Muitas vezes por ausência de conhecimento sobre a importância da prevenção e/ou falta da orientação do profissional que o acompanha (LIBERATO SMD, 2017).

Dessa forma, é evidente a necessidade de traçar estratégias de prevenção, transformação no estilo de vida e atenção aos fatores de risco preveníveis para essas lesões (tabagismo, etilismo, obesidade, uso correto de anti-hipertensivos, dentre outros) e orientações aos pacientes.

A lesão crônica de perna relacionada com hipertensão venosa dos membros inferiores corresponde a uma alta variação em úlceras encontradas nos membros inferiores, responsáveis por dor e interferência na mobilidade (LIMA BE, et al., 2018). Entre as lesões agudas e crônicas, localizaram-se mais frequentemente nos pés $D / E(10 / 75,00 \%)$ dos participantes da pesquisa, causando transtornos físicos e alteração da marcha, piora da doença de base ou prejuízo para o tratamento, impactando na qualidade de vida. Um importante achado durante o exame físico nos membros inferiores em pacientes portadores de lesões crônicas e agudas, é a identificação de possíveis obstruções arteriais e/ou variação da perfusão tissular dos membros. A alteração dos pulsos dorsal do pé e tibial posterior é indício de menor vascularização da lesão, consequentemente os membros inferiores e a cor da pele ao redor da ferida apresentarão hipopigmentação $(5 / 41,67 \%)$.

O estudo revelou que no ambulatório de lesões cutâneas a mediana da idade dos pacientes que foram admitidos no período em que foi realizada o estudo é de 72 e a média é de 74,5. Em idosos, a pele torna-se mais vulnerável ao aparecimento de lesões em decorrência das modificações vasculares, metabólicas e imunológicas que acometem esta faixa etária (CHIBANTE C, et al., 2015). 
Sachett JAG e Montenegro CS (2019) observaram em seu estudo epidemiológico dos pacientes atendidos pelo programa "Melhor em Casa", a prevalência de lesões em membros inferiores e, dentre estas lesões, 0 diabetes melito e a hipertensão arterial foram relatados como comorbidades associadas à cronificação das lesões e ao retardo na cicatrização. Outro estudo em um Ambulatório de cuidados com feridas demostrou que os pacientes tinham como principais comorbidades a Hipertensão arterial seguida da junção entre a hipertensão arterial e o Diabetes mellitus (SACHETT JAG e MONTENEGRO CS, 2019; SQUIZATTO RH, et al., 2017).

Acerca das limitações do estudo, a primeira limitação presente foi a quantidade pequena da amostra pois apesar de ser ambulatório em hospital público, o atendimento era feito apenas com encaminhamento médico e por uma enfermeira estomaterapeuta. Segunda limitação, em muitas situações os pacientes faltaram a consulta com a enfermeira e, consequentemente, não se realizava o curativo, logo a coleta era realizada em outro momento.

O Ambulatório onde o estudo foi realizado atende pacientes com diferentes tipos de feridas, como lesões pós-cirúrgicas, traumáticas, queimaduras e feridas crônicas e, apesar do tamanho da amostra, os dados evidenciados apresentam grande relevância pois possibilitou conhecer as características da população atendidas em um Ambulatório de referência para o tratamento de lesões, permitindo direcionar as ações de cuidado no manejo com feridas com uma abordagem biopsicossocial, considerando-se as peculiaridades da população com lesões agudas e crônicas no contexto amazônico e seus determinantes sociais. Os dados apresentados são significativos pois endossam outros estudos nacionais que demonstraram a predominância de pacientes do sexo masculino, idosos, presença de comorbidades como Diabetes e Hipertensão arterial e o acometimento dos membros inferiores, podendo fundamentar as políticas públicas de saúde adotadas no Brasil.

Por fim, evidencia-se a relevância do conhecimento das diretrizes clínicas, consensos e níveis de evidências que norteiam o cuidado de lesões agudas e crônicas, em especial as medidas de prevenção e 0 autocuidado, sendo assim, essa pesquisa, apesar de regional, contribui fortemente para o aprimoramento do cuidado com lesões.

\section{CONCLUSÃO}

A realização desta pesquisa revelou achados importantes quanto à correlação do meio sociodemográfico com os aspectos clínicos das feridas agudas e crônicas. O tratamento de lesões requer especial envolvimento dos profissionais da saúde, dentre eles, o enfermeiro destaca-se como parte fundamental no processo de cura e fechamento das lesões, portanto, deverá obter entendimento necessário para embasar a sua prática em pesquisas como esta que apresentou a situação sociodemográficas e clínica dos portadores de lesões em um ambulatório de Manaus.

\section{REFERÊNCIAS}

1. ALVES DFS, et al. Translation and Adaptation of The Bates-Jensen Wound Assessment Tool for The Brazilian Culture. Texto contexto - enferm., Florianópolis, 2015; 24(3): 826-833.

2. AZEVEDO IC, et al. Perfil de la producción científica de enfermería nacional sobre heridas. Rev Cubana Enferm, 2018; 34(1).

3. CHAVAGLIA SRR, et al. Caracterização de pacientes com lesão cutânea em unidades de internação médica e cirúrgica. Rev Enferm UFPE, 2015; 9(1): 183-92.

4. CHIBANTE C, et al. Perfil de clientes hospitalizados com lesões cutâneas. Revista Cubana de Enfermería, 2015; 31(4).

5. GALVÃO NS, et al. Aspectos epidemiológicos e clínicos de pacientes com úlcera por pressão internados em uma instituição hospitalar, Manaus, Revista Estima; 2015; 13 (3): 91-96.

6. GALVẢO NS. Prevalência de feridas agudas e crônicas e fatores associados em pacientes de hospitais públicos em Manaus-AM. Tese (Doutorado em enfermagem na saúde do adulto). Escola de Enfermagem, Universidade de São Paulo, São Paulo, 2016.

7. GRDEN CRB, et al. Lesões de pele em idosos hospitalizados. Revista Estima, 2018; 16(4118).

8. LIBERATO SMD, et al. Adesão ao tratamento de pessoas com úlceras venosas atendidas na atenção primária à saúde. Aquichan, 2017; 17(2): 128-139.

9. LIMA BE, et al. Prevalence of Chronic Wounds in A City of Minas Gerais (Brazil). REME: Revista Mineira de Enfermagem, 2018; 22(1143). 
10. LIMA LV, et al. Conhecimento de Pessoas com Úlceras Vasculogênicas acerca da Prevenção e dos Cuidados com as Lesões. Revista Estima, 2016; 11(3).

11. LIMA NBA, et al. Perfil sociodemográfico, clínico e terapêutico de pacientes com lesões agudas e crônicas. Rev Enferm UFPE online. Universidade Federal de Campina Grande/UFCG, 2016; 10(6): 2005-2017.

12. MACEDO EAB, et al. Knowledge of the care of ounded patients: evidence of validity of an instrument. Rev Bras Enferm, 2019; 72(6): 1562-70.

13. MELO AF, et al. Construction, validation and reliability of an instrument for evaluation and evolution of chronic wounds. Biosci. J, 2019; 35(4).

14. MITTAG BF, et al. Cuidados com Lesão de Pele: Ações da Enfermagem. Revista Estima, 2017; 15(1): $19-25$.

15. OLIVEIRA AC, et al. Qualidade de vida de pessoas com feridas crônicas. Acta Paul Enferm, 2019; 32(2): $194-201$.

16. SACHETT JAG, MONTENEGRO CS. Perfil epidemiológico dos pacientes atendidos pelo programa "Melhor em Casa". Revista Estima, 2019; 17(737).

17. Squizatto $\mathrm{RH}$, et al. Perfil dos usuários atendidos em ambulatório de cuidado com feridas. Cogitare Enfermagem, 2017; 22(1).

18. VIEIRA CPB, ARAUJO TME. Prevalência e fatores associados a feridas crônicas em idosos na atenção básica. Rev. esc. enferm. USP, 2018; 52(03415).

19. VIEIRA CPB, et al. Prevalência e caracterização de feridas crônicas em idosos assistidos na atenção básica. Rev Baiana Enferm, 2017; 31(3): 17397. 